\title{
LA LEGISLACIÓN A FAVOR DE LA ADOLESCENTE EMBARAZADA ${ }^{1}$
}

\author{
LEGISLATION IN FAVOR OF PREGNANT ADOLESCENT \\ Miriam Helena Jaramillo Jimenez $z^{2}$, Juliana Zarate Cedano ${ }^{3}$, \\ Ligia Betty Arboleda 4 , Alexandra Marieth Barajas Blanco ${ }^{5}$
}

\section{RESUMEN}

Introducción: La legislación y las políticas son herramientas necesarias para lograr el desarrollo y la evolución en todos los aspectos de un país. Para el profesional de enfermería la creación de estrategias de cuidado y mejoramiento depende del conocimiento que tenga sobre estos lineamientos y de la formación académica, logrando aportar como ciencia independiente disminución de problemáticas como es el embarazo adolescente. Materiales y Métodos: Mediante una búsqueda de literatura y el análisis de la legislación en Colombia se realiza una reflexión sobre la situación de la adolescente embarazada. Resultados: Se logra evidenciar que en Colombia y el mundo existen herramientas legislativas y estrategias de apoyo a los niños, niñas y adolescentes, y están en búsqueda de disminuir la situación de embarazos a temprana edad. Así mismo se destaca a la Enfermera eje fundamental en la salud de la población como ente educador. Discusión y Conclusiones: Es de suma importancia que la Enfermera(o) no limite la consulta o la atención a la necesidad del momento sino que tenga un alcance más amplio, que tenga una visión de la persona identificando necesidades propias de la edad y apoyada en las políticas y legislación del país realizar un cuidado integral. Se recomienda que haya más flujo de información de los derechos sexuales y reproductivos en toda la población, donde se destaquen aspectos como proyecto de vida y refuerzo de valores. (Rev Cuid 2012; 3(3):419-23).

Palabras clave: Política de Salud. Legislación de Enfermería. Adolescente. (Fuente: DeCS BIREME).

\section{ABSTRACT}

Introduction: The law and policy are necessary tools for development and evolution in all aspects of a country. For the nurse creation care and improvement strategies depends on the knowledge you have about these guidelines and academic training, managing to provide independent science decline as problematic as teenage pregnancy. Materials and Methods: A search of literature and an analysis of the laws in Colombia as a reflection on the situation of pregnant adolescents. Results: We show that succeeds in Colombia and the world there are legislative tools and strategies to support children and adolescents, and are seeking to diminish the status of early pregnancy. It also highlights the fundamental axis Nurse health of the population as being an educator. Discussion and Conclusions: It is extremely important that the nurse does not limit the query or the attention to the need of the moment but have a broader scope, with a vision of the person identifying needs of the age and supported in policies and legislation in the country to perform a comprehensive care. It is recommended that more flow of information on sexual and reproductive rights entire population, where as a project highlighting aspects of life and reinforce values.

Key words: Health Policy. Legislation, Nursing. Adolescent. (Source: DeCS BIREME).

1 Articulo de Reflexión.

2 Enfermera Egresada Universidad de Santander UDES. Asistente de Investigación, Grupo de Investigación de EnfermeríaEverest. Integrante Grupo de Cuidado, Programa de Enfermería UDES.

3 Enfermera Egresada Universidad de Santander UDES. Integrante Grupo de Cuidado.

4 Enfermera Egresada Universidad Industrial de Santander. Especialista en Docencia Universitaria. Magistrada del Tribunal Departamental Ético de Enfermería de Santander. Miembro de la Sigma Theta Tau International - Honor Society of Nursing. Miembro del Grupo de Cuidado, Integrante del Grupo Multicéntrico, Programa de Enfermería UDES.

5 Estudiante X Semestre, Integrante Grupo de Cuidado, Programa de Enfermería UDES. Articulo recibido el 01 de Octubre de 2012 y aceptado para su publicación el 22 de Noviembre de 2012. 


\section{INTRODUCCIÓN}

Es indispensable para el profesional de enfermería conocer la legislación de su país, y poder enfrentar las diferentes problemáticas sociales y complementado con su formación profesional el desarrollo de estrategias de mejoramiento frente al embarazo adolescente. A nivel mundial existe mucho interés en disminuir esta situación, es así como la Organización Mundial de la Salud y la Organización de las Naciones Unidas, la incluyeron dentro de los objetivos del milenio específicamente en el número cinco "mejorar la salud sexual y reproductiva", dentro de este objetivo esta una meta universal que es disminuir la mortalidad materna, y a su vez Colombia tiene como meta detener el crecimiento del porcentaje de adolescentes que han sido madres o están embarazadas, manteniendo esta cifra por debajo de $15 \%(1,2)$. Para el 2010 esta cifra según la Encuesta Nacional de Demografía y salud (ENDS) (3) muestra un estado de 19.5\%, lo que es preocupante, si tenemos en cuenta que solo ha disminuido desde el año 2005 en un punto porcentual según el observatorio de asuntos de genero de Colombia (4). En Nuestro país encontramos diversos escritos legislativos, fundamentalmente la Constitución Política de Colombia de 1991, ley 115/1994, ley 1098/2006, ley $1146 / 2007$, decreto $3039 / 2007$, ley $1257 / 2008$, decreto 2968/2010, que aplicadas según el hecho, disminuirían la problemática y orientaría al cuidador. Como lo afirman en el documento CONPES social 140 (2012) (5). Con este trabajo se pretende recopilar y resumir de forma práctica todos estos estatutos, para que sea una herramienta de fácil acceso para el profesional de enfermería enfrentado a estos casos y tenga herramientas para brindar educación y pueda servir de guía para las familias implicadas.

\section{Los embarazos en adolescentes}

El embarazo en adolescente más que un problema de salud pública afecta el desarrollo del país, pues detrás de esto hay una cadena de acontecimientos que retrasan el progreso educativo, económico y social y afecta de manera adversa el desempeño socioeconómico de las madres y las condiciones de salud y nutrición de los niños, y constituye un obstáculo para la movilidad social y la superación de la pobreza (6). Los embarazos adolescentes, por ejemplo, explican casi 10 por ciento de todos los casos de deserción escolar entre las mujeres menores de veinte años (7). En viceversa la pobreza y desigualdades en el acceso a la educación perpetúan los altos porcentajes de esta problemática poniendo en peligro la salud de las jóvenes y limitando sus oportunidades de prosperidad social. Así que es un ciclo vicioso donde ninguna de las dos partes puede avanzar (8). No hay que desconocer los contextos en los que cursa esta problemática, la Encuesta de Demografía y Salud muestra varios puntos importantes como la exposición al riesgo de embarazo, demanda insatisfecha de planificación familiar, bajo nivel de escolaridad y área rural como más vulnerable, lo que a nuestra profesión concierne hay falta de educación e información por parte del profesional a la población afectada que posiblemente sea por falta de conocimientos y fortaleza en dicha área (3).

La presente reflexión es basada en la revisión general de la legislación en Colombia que compete la atención a la adolescente embarazada y su entorno, que permita que el profesional de enfermería brinde un cuidado integral de forma efectiva y eficaz.

¿Qué políticas existen para disminuir esta situación?

\section{Figura 1. De los Objetivos del Milenio}

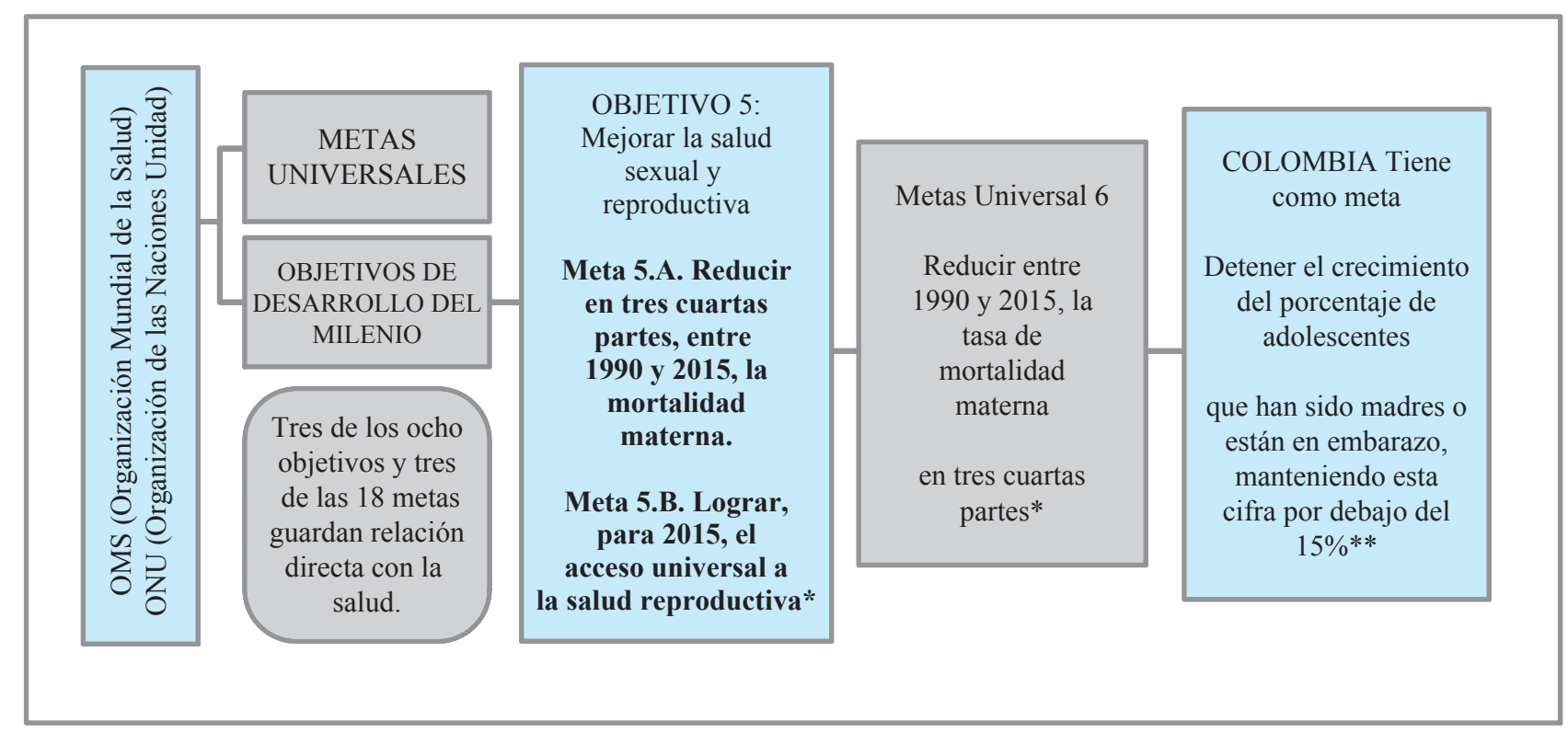


De acuerdo con las proyecciones de población del Departamento Administrativo Nacional de Estadística (DANE) para este año, Colombia es un país con una población de más 13 millones de niños, niñas y adolescentes. De los cuales, 4,3 millones se encuentran entre los 5 y los 9 años; 4,3 millones entre los 10 y los 14 años y 4,4 entre los 15 y los 19 años, de tal forma que el $28 \%$ de la población total en el país corresponde al grupo entre los 5 y los 19 años, teniendo en cuenta estos datos, es grande la población a intervenir para que Colombia cumpla con la meta planteada hace ya más de una década. Colombia (9).

El Departamento Nacional de Planeación ha diseñado lineamientos para el desarrollo de una estrategia para la prevención del embarazo en la adolescencia y la promoción de proyectos de vida para los niños, niñas adolescentes y jóvenes en edades entre 6 y 19 años, publicado como el Documento CONPES social No 147, donde fortalece la importancia de este problema nacional fundando para la sociedad, lineamientos para la creación de capital social; promoviendo factores protectores, en la toma de decisiones responsables informadas sobre el cuerpo humano, los derechos sexuales y Reproductivos (10). Con el propósito de prevenir el embarazo en adolescentes, involucrando a sectores sociales como redes de jóvenes, las fuerzas militares, la policía, medios de comunicación, programas de ICBF. Reconociendo como eje principal la familia, su funcionalidad en la comunidad y el desarrollo de un proyecto de vida.

Por otra parte es importante mencionar que en nuestro país encontramos una entidad importantísima que siendo privada, causa gran impacto en la salud Colombiana como lo es Profamilia y ¿Qué es Profamilia? La Asociación Probienestar de la Familia Colombiana Profamilia, es una entidad privada sin ánimo de lucro especializada en salud sexual y salud reproductiva que ofrece servicios médicos, educación y venta de productos a la población colombiana (11). Dentro de sus programas se encuentra Profamilia Joven donde se encuentran personas expertas que mediante asesorías gratuitas e individuales proporcionan respuestas acertadas a dudas y necesidades en materia de salud sexual o salud reproductiva para que los jóvenes entre 13 y 21 años puedan tomar decisiones acertadas e informadas, además, ofrece asesoría y capacitación en temas relacionados con sexualidad, salud sexual y reproductiva y promoción de derechos sexuales y reproductivos a padres y madres, docentes y personal del sector educativo y de salud (12).

Es de suma importancia que el profesional de enfermería conozca que hay ofertado para los jóvenes, y remitirlos a estos sitios, no limitar la consulta o la atención de enfermería a la necesidad del momento sino que tenga un alcance más amplio, que se tenga una visión de la persona que se está atendiendo identificando necesidades propias de la edad y realizar un cuidado integral. No podemos caer en el error de pensar que porque la joven ya está embarazada, no hay que hablarle de Planificación, con mayor razón hay que hacerle intervención de promoción y prevención, sobre todo hay que priorizar la educación en estas madres jóvenes.
Todas estas políticas están basadas en la legislación y sirven como soporte para la creación de estrategias de mejoramiento, a continuación nombraremos las que a nuestro parecer son las más relevantes, ya que destacan los derechos más importantes y que están basados en los derechos humanos, como el derecho a la vida, la integridad, la individualidad, al respeto, a la salud entre otros. Como principal soporte se encuentra la Constitución política de Colombia, dentro de esta resaltanlos derechos de los niños, los cuales prevalecen sobre los demás derechos. Así mismo enfatiza que el adolescente tiene derecho a la protección y a la formación integral. El Estado y la sociedad garantizan la participación activa de los jóvenes en los organismos públicos y privados que tengan a cargo la protección, educación y progreso de la juventud (13).

Del mismo modo es interesante examinar también en esta problemática social el Código de la Infancia y la Adolescencia Ley 1098 de 2006: Cuyo propósito es garantizar a los niños, a las niñas y a los adolescentes su pleno y armonioso desarrollo para que crezcan en el seno de la familia y de la comunidad, en un ambiente de felicidad, amor y comprensión. Prevalecerá el reconocimiento a la igualdad y la dignidad humana, sin discriminación alguna. En este establecen que se entiende por protección integral de los adolescentes el reconocimiento como sujetos de derechos he instituye obligaciones especiales del sistema de seguridad social en salud, como: diseñar, desarrollar y promocionar programas que garanticen a las mujeres embarazadas, el tratamiento y el cuidado y atención para evitar durante el embarazo, parto y posparto la transmisión vertical madre-hijo del VIH/SIDA. Garantizar atención oportuna y de calidad a todos los adolescentes, en especial en los casos de urgencias. Garantizar la actuación inmediata del personal médico y administrativo cuando un adolescente se encuentre hospitalizado o requiera tratamiento o intervención quirúrgica y exista peligro inminente para su vida, y desarrollar programas de apoyo prioritario a las madres adolescentes (14).

Teniendo en cuenta el incremento de las tasas de fecundidad de esta población en las últimas décadas, hacen necesario sensibilizar a la sociedad colombiana sobre los factores de riesgo que inciden en la problemática del embarazo en adolescentes y sus consecuencias personales, familiares y sociales, destacando la corresponsabilidad de la familia, la sociedad y el Estado en su atención, cuidado y protección; es así como mediante el Decreto 3705 de 2007 declara como "Día Nacional de la Prevención del Embarazo en Adolescentes" el 26 de septiembre de cada año (15).

Por otro lado en el Decreto 2968 de 2010, por el cual se crea la Comisión Nacional Intersectorial para la promoción y garantía de los derechos sexuales y reproductivos y establecen los espacios de participación y los actores involucrados en la planeación, ejecución, seguimiento y evaluación de los programas, estrategias y acciones que sean orientadas al logro de los Objetivos del Desarrollo del Milenio, la garantía de los Derechos Sexuales y Reproductivos y el Desarrollo Integral de la 
población colombiana (16).

No hay que desconocer que la problemática hay que abarcarla desde todos los entes es así como la ley 115 de 1994, que tiene por objeto la educación como un proceso de formación permanente, personal, cultural y social que se fundamenta en una concepción integral de la persona humana, de su dignidad, de sus derechos y sus deberes. Mediante esta ley se busca desarrollar una sana sexualidad que promueva el conocimiento de sí mismo y la autoestima, la construcción de la identidad sexual dentro del respeto por la equidad de los sexos, la afectividad, el respeto mutuo y prepararse para una vida familia armónica y responsable (17).

Todos estos estatutos apoyan las políticas de proyección social para una población tan vulnerable como son los niños, niñas y adolescentes.

\section{CONCLUSIONES}

La importancia que el profesional de enfermería conozca los sistemas de salud y las leyes, le permite tener la capacidad de orientar a la embarazada, familia y comunidad para la utilización de los mismos, así mismo se fortalece la disciplina ya que aumenta la credibilidad ante la población la cual encuentra en la enfermera un profesional competente en áreas sensibles para los individuos.

El embarazo adolescente es un problema que requiere más educación y apoyo por parte del estado permitiendo que los jóvenes adquieran mayor responsabilidad para enfrentar esta situación con mayor conocimiento en una edad adecuada.

Es importante que por parte de los profesionales de salud haya mayor flujo de información sobre derechos sexuales y reproductivos en toda la población, donde se destaquen aspectos como proyecto de vida y refuerzo de valores.

Ampliar la visión de este tema, para que además de verse como un problema de salud pública se mire desde el punto de vista de desarrollo del país. 


\section{REFERENCIAS BIBLIOGRÁFICAS}

1. Organización Mundial de la Salud (OMS). Objetivos de desarrollo del milenio. Disponible en: http://www.who. int/topics/millennium_development_goals/es/index.html Consultado: Septiembre 3,2012.

2. Buenas Prácticas en lo Local. Promoción de la Salud y los Derechos Sexuales y Reproductivos en los Montes de María Secretaria General, 2005: 48-55. Disponible en: http://www.pnud.org.co/img_uplo ad/33323133323161646164616461646164/Mejorar\%201a\%20salud\%20sexual\%20y\%20reproductiva.pdf

3. Ministerio de la Protección Social. Encuesta Nacional de Demografía y Salud. Disponible en: http://www. profamilia.org.co/encuestas/Profamilia/Profamilia/index.php?option $=$ com_content\&view=article\&id=62\&Item id=9 Consultado: Agosto 29,2012.

4. República de Colombia. Seguimiento a la política social del gobierno e indicadores poblacionales, con enfoque de género informe de la Alta consejería presidencial para la equidad de la mujer. Bogotá: República de Colombia; 2011. Disponible en: http://www.equidadmujer.gov.co/OAG/Documents/Salud-Estado-Embarazo-maternidad-Adolecentes.pdf Consultado: Septiembre 2, 2012.

5. Departamento Nacional de Planeación. Indicadores CONPES Social. Documento Conpes Social n 140 . (14 de Junio 2005). Disponible en: http://www.dnp.gov.co/Programas/DesarrolloSocial/Pol\%C3\%ADticasSocialesTra nsversales/ObjetivosdeDesarrollodelMilenio.aspx Consultado: Agosto 30, 2012.

6. Gaviria A, Palau MDM. Nutrición y salud infantil en Colombia: determinantes y alternativas de política. [ Revista en línea] 2006[ Consultado: 28 Septiembre de 2012]; 36(2): 1-36. Disponible en: http://agaviria.uniandes. edu.co/papers_pub/Nutrition_and_Infant_Health_in_Colombia.pdf

7. Gaviria A. Increasing Returns and the Evolution of Violent Crime: The Case of Colombia.[ Revista en linea] 2000[ Consultado: 28 septiembre de 2012]; 61(2000): 1-25. Disponible en: http://www.sciencedirect.com/science/article/pii/S0304387899000590

8. Naciones Unidas. Objetivos de desarrollo del milenio, 2010. Disponible en: http://www.un.org/spanish/millenniumgoals/pdf/MDG_Report_2010_SP.pdf\#page=34 Consultado: Septiembre 18,2012.

9. Instituto de Bienestar Familiar (ICBF). Lineamiento técnico del programa de promoción y prevención para la protección integral de niños, niñas y adolescente. Disponible en: http://www.icbf.gov.co/portal/page/portal/ Descargas1/LineamientosPPPINNAMayo9de2012.pdf Consultado: Septiembre 15,2012.

10. Departamento Nacional de Planeación. Lineamientos para el desarrollo de una estrategia para la prevención del embarazo en la adolescencia y la promoción de proyectos de vida para los niños, niñas, adolescentes y jóvenes en edades entre 6 y 19 años. Documento Conpes Social n 147. (31 Enero de 2012). Disponible en: http://www. dnp.gov.co/LinkClick.aspx?fileticket=BSINpiuEDQ0\%3D\&tabid=1473 Consultado: Septiembre 26, 2012.

11. Profamilia. La Asociación Probienestar de la Familia Colombiana. Disponible en: http://www.profamilia.org.co Consultado: Septiembre 6,2012.

12. Profamilia. Profamilia Joven, 2012. Disponible en: http://www.profamiliajoven.org.co/index.php?option=com content $\&$ view $=$ article $\&$ id $=124$ : profamiliajoven $\&$ catid $=35:$ conocenos $\&$ Itemid $=80$. Consultado: Septiembre 16,2012 .

13. Constitución política de Colombia, (1991). Disponible en: http:/www.encolombia.com/derecho/Constitucion Colombia/Contenido.htm . Consultado: Septiembre 17,2012.

14. Código de Infancia y Adolescencia. Ley 1098 / 2006 de 8 de noviembre. Diario Oficial $\mathrm{n}^{\circ}$ 46446. (8 de mayo de 2007). Disponible en: http://www.alcaldiabogota.gov.co/sisjur/normas/Norma1.jsp?i=22106

15. La Prevención Del Embarazo En Adolescentes. Decreto 3705/2007 de 24 de septiembre. Diario Oficial 46762 del Ministerio de la Protección Social. ( 25 septiembre de 2007)

16. Para la promoción y garantía de los derechos sexuales y reproductivos. Decreto 2968 / 2010 de 6 de agosto. Boletín oficial del Ministerio de la Protección. (6 agosto de 2010).

17.Ley General de Educación. Ley 115/ 1994 de 8 de febrero. Diario Oficial nº41.214. (8 de febrero de 1994). Disponible en: http://www.alcaldiabogota.gov.co/sisjur/normas/Norma1.jsp?i=22106 